
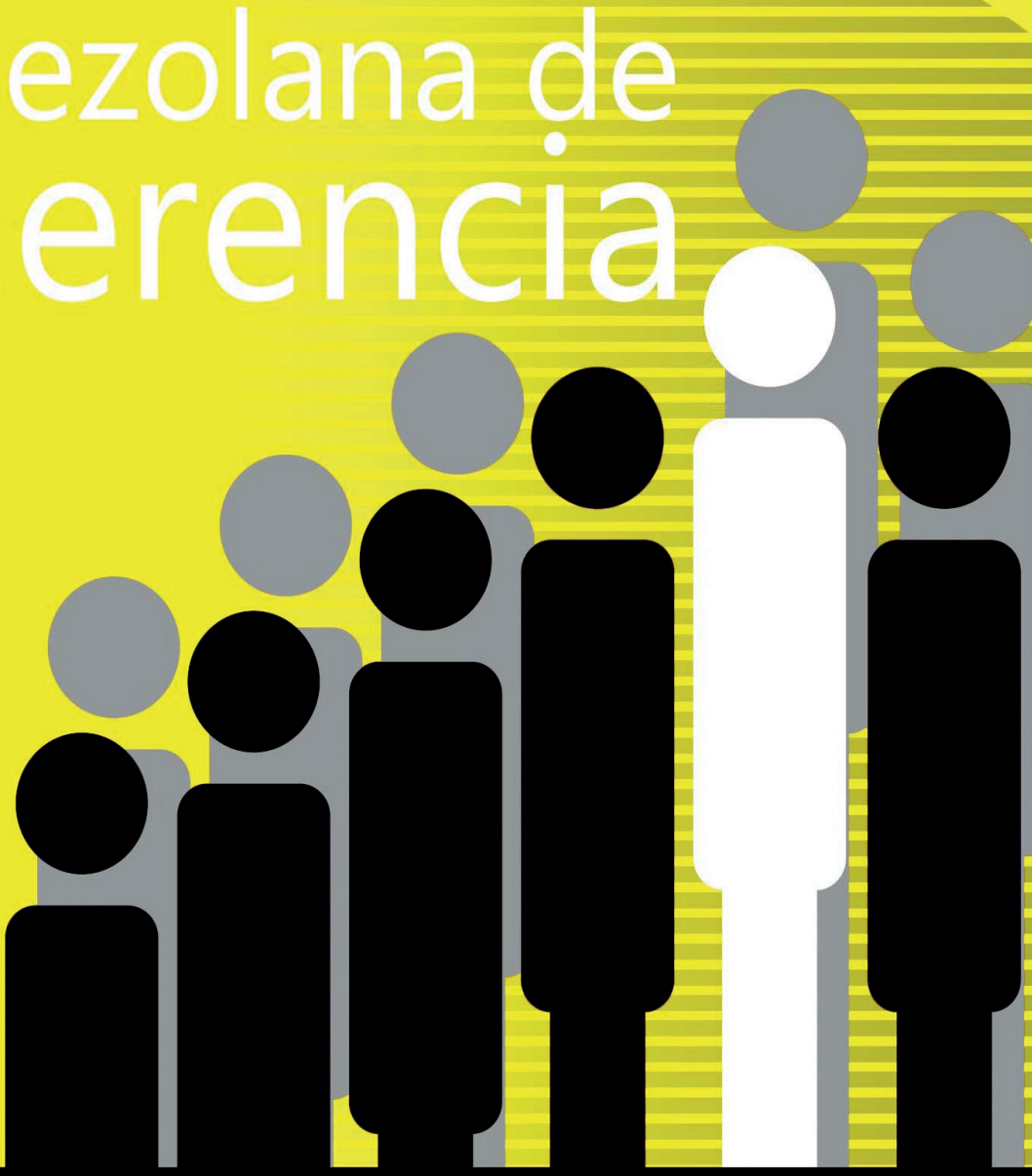


\title{
Teletrabajo en Sudamérica: Un desafío jurídico frente al covid-19
}

\author{
Ramírez Velásquez, Joanna Carolina* \\ Tapia Tapia, Héctor Eduardo* \\ Vega Abad, Cesar Remigio ${ }^{* \star}$ \\ Villagómez, Martha
}

\begin{abstract}
Resumen
El teletrabajo es un modo de prestación de servicios bajo relación de dependencia, fuera del lugar habitual de trabajo y con el uso esencial de medios tecnológicos. En Sudamérica su implementación está regulada en alguno estados mediante ley o resoluciones ministeriales y en otros sin norma específica que lo establezca. El objetivo del presente estudio fue examinar la legislación establecida para la implementación del teletrabajo frente al Covid-19 en Sudamérica. La metodología consistió en la revisión bibliográfica y documental de la literatura y legislaciones establecidas para la implementación del teletrabajo. Para la recolección de la información se utilizaron las bases de datos: Redalyc, Latindex, Scielo, Dialnet, DOAJ, SCOPUS, así como otras fuentes oficiales nacionales e internacionales. La pandemia causada por el Covid-19 ha provocado una crisis en el mercado laboral que obliga a la implementación del teletrabajo generando urgentes pronunciamientos gubernamentales. Se evidencia en Sudamérica su gran impulso como medio alternativo para enfrentar la emergencia socio-laboral y económica, algunos estados desarrollaron su normativa y otros avanzaron en promover su práctica.
\end{abstract}

Palabras Clave: Teletrabajo; mercado laboral; legislación laboral; Covid-19.

Recibido: 18.09.20 Aceptado: 20.01.20

* Magíster Scientiarum en Derecho Laboral y Administración del Trabajo. Master en Salud de los Trabajadores. Docente de tiempo completo de la Universidad Católica de Cuenca. Extensión San Pablo de la Troncal. Ecuador. Correo electrónico: joanna.ramirez@ucacue.edu.ec. ORCID: https://orcid.org/0000-0003-32667195

** Magíster en Derecho Constitucional. Magister en Derecho Administrativo. Especialista en Tributación. Director de la Carrera de Derecho de la Universidad Católica de Cuenca. Extensión San Pablo de la Troncal. Ecuador. Correo electrónico hector.tapia@ucacue.edu.ec. ORCID: https://orcid.org/0000-0003-1763-8302

*** Magíster en Redes de comunicaciones. Docente de tiempo completo de la Universidad Católica de Cuenca. Extensión San Pablo de la Troncal. Ecuador. Correo electrónico: crvegaa@ucacue.edu.ec ORCID: https:// orcid.org/0000-0003-3301-2668

**** Estudiante de la Carrera de Derecho. Universidad Católica de Cuenca. Extensión San Pablo de la Troncal. Ecuador. 


\title{
Teleworking in South America: A legal challenge against covid-19
}

\begin{abstract}
Teleworking is a way of rendering services under a relationship of dependence, outside the usual place of work and with the essential use of technological means. In South America its implementation is regulated in some states by law or ministerial resolutions and in others without specific rule that establishes it. The objective of the present study was to examine the legislation established for the implementation of telework against Covid-19 in South America. The methodology consisted of a bibliographic and documentary review of the literature and legislation established for the implementation of telework. For the collection of information were used databases: Redalyc, Latindex, Scielo, Dialnet, DOAJ, SCOPUS, as well as other national and international official sources. The pandemic caused by the Covid-19 has caused a crisis in the labor market that forces the implementation of teleworking generating urgent governmental pronouncements. It is evident in South America its great momentum as an alternative means to address the socio-labor and economic emergency, some states developed their regulations and others advanced in promoting their practice.
\end{abstract}

Key Words: Teleworking; labor market; labor legislation; Covid-19

\section{Introducción}

El teletrabajo consiste en el desempeño de funciones laborales, con el uso de los medios tecnológicos, sin requerirse la presencia física del trabajador o trabajadora en un lugar específico u oficina del empleador.

En América Latina producto del avance de las tecnologías de la información y la comunicación el teletrabajo comenzaba a implementarse en algunos sectores; según la Academia Internacional de Teletrabajo para América Latina y el Caribe (2017) en Brasil estiman que el porcentaje de teletrabajadores es de $16,2 \%$, y en Chile comenzó su aplicación en el Instituto Nacional de Propiedad Intelectual en los años 2017-2018 alcanzando hasta el $10 \%$ del personal de servicio. En el Ecuador de acuerdo a los indicadores laborales del Ministerio del Trabajo (2020) el teletrabajo emergente entre el mes de marzo a octubre de 2020 alcanzó en el sector público 286.401 trabajadores y en el sector privado 155.208.

Por otra parte, de acuerdo al Ministerio de Producción y Trabajo (2019) en Argentina para el año 2017 el número de teletrabajadores fue de 267.826 , lo que representa un $7,8 \%$. Y en Colombia para el 2018 la cifra de teletrabajadores alcanzó 122.278 en donde Bogotá, Medellín y Cali concentran el $87,7 \%$ (Tapasco-Alzate y García-Giraldo, 2020).

De este modo, aun cuando algunos 
países de Sudamérica comenzaban a desarrollar con éxito su implementación; no en todos se encuentra regulado mediante Ley, en Ecuador el teletrabajo inicia según lo expuesto por el Ministerio del Trabajo (2016) mediante Acuerdo Ministerial No. MDT-2016-190, en el caso de Colombia está contemplado su implementación tal como lo prevé el Congreso Nacional (2008) mediante la Ley número 1221 de 2008, y en Perú está regulado de acuerdo a lo estipulado por el Congreso de la República de Perú (2013) en la Ley 30.036 de 2013. No obstante, Venezuela no cuenta con una Ley que establezca el teletrabajo expresamente, empleándose según la Asamblea Nacional de la República Bolivariana de Venezuela (2012) en la Ley Orgánica del Trabajo, Los Trabajadores y Las Trabajadoras (2012).

En Argentina su implementación estaba sujeta principalmente a las disposiciones de la Ley del Contrato del Trabajo Nro. 20744 y el Convenio 177 de la Organización Internacional del Trabajo, ya que desde el año 2007 el Ministerio del Trabajo, Empleo y Seguridad Social elaboró un Proyecto de Ley del Teletrabajo el cual perdió estado legislativo (Ministerio de Producción y Trabajo, 2019).

Bajo este contexto, cabe destacar que ante la declaración de emergencia de salud pública declarada por la Organización Mundial de la Salud (2020) por el brote del nuevo coronavirus (2019-nCoV) que llevó a dictar medidas de aislamiento en la población, entre estas, la suspensión de las jornadas laborales presenciales, se presenta el teletrabajo como principal alternativa, constituyendo un desafío en Sudamérica debido al poco desarrollo que existía en su implementación y a la falta de una regulación jurídica expresa, lo que obligó a los estados a la promulgación urgente de resoluciones que regularan el teletrabajo emergente garantizado los derechos laborales y manteniendo las relaciones contractuales.

Ante lo expuesto, es menester el abordaje de esta figura jurídica, que aun cuando se inicia en Sudamérica, formará parte esencial del desarrollo económico de la región frente a la pandemia y posterior a ella, por ello, surge el proyecto de investigación titulado: "Análisis jurídico sobre la legislación establecida para la implementación del teletrabajo frente al Covid-19 en Sudamérica. Una revisión." De la Universidad Católica de Cuenca. Unidad Académica de Ciencias Sociales, Extensión La Troncal, con el fin de generar nuevos conocimientos sobre el teletrabajo, que realicen un aporte desde la siguiente interrogante ¿Qué nuevas perspectivas presenta la legislación establecida para la implementación del teletrabajo frente al Covid-19 en Sudamérica? para responder a la interrogante, esta investigación se planteó como primer objetivo que desarrolla el proyecto en curso: Examinar la legislación establecida para la implementación del teletrabajo frente al Covid-19 en Sudamérica.

La metodología utilizada consistió en la revisión bibliográfica y documental de la literatura, reportada a través de los artículos indexados en las principales bases de datos: Redalyc, Latindex, Scielo, Dialnet, DOAJ, SCOPUS, JSTOR, durante los últimos cinco años, y documentos oficiales nacionales e internacionales que trataran el teletrabajo en Sudamérica frente a la pandemia, aunado a la revisión exhaustiva de las diferentes legislaciones que regulan el teletrabajo en Venezuela, Colombia, Ecuador, Perú, Chile, Argentina, Bolivia, 
Uruguay, Paraguay y Brasil, los criterios utilizados para la búsqueda fueron: Teletrabajo en Latinoamérica, teletrabajo emergente, legislación sobre teletrabajo.

\section{Antecedentes del teletrabajo}

La evolución de las tecnologías de comunicación e información han producido grandes avances en los modos de prestación de servicio y en consecuencia el surgimiento del teletrabajo. Su implementación tiene origen en la crisis petrolera de 1970, que conllevó el alza del petróleo y el incremento en los costos de traslado diario de los trabajadores de sus domicilios al lugar de trabajo, de esta forma se planteó como solución a la problemática, donde los trabajadores prestarian sus servicios desde el domicilio para evitar la utilización del transporte y en consecuencia gastos de traslado (Organización Internacional del Trabajo, 2016).

Al respecto Fernández (2019:35) refiere: En esa época, existían computadoras e internet, su uso en Estados Unidos se hizo generalizado sólo dos décadas después. En un inicio, el teletrabajo sólo era para aquellos altos ejecutivos que tenían el privilegio de trabajar desde sus hogares. Sin embargo, debido a los resultados positivos en su aplicación y la competitividad presente en el mercado laboral esta nueva herramienta de trabajo se torna de interés para el grupo empresarial. Para Culqui \& González (2016:96) en principio fue concebida para hacer frente a una crisis energética específica, fue convirtiéndose en una alternativa que daría solución a diversos problemas sociales, personales y organizacionales.

En el caso de América Latina, desde fines del siglo $X X$ se ha iniciado la implementación del teletrabajo, en atención a la evolución tecnológica de la información y la comunicación, además del acelerado desarrollo de los procesos productivos, la aceptación de los empleados y la reducción de los costos vinculados con los medios para trasporte, vestimenta, alimentos, y combustibles (Benjumea-Arias et al, 2016). Es claro que en la región el uso de las herramientas digitales ha generado grandes transformaciones en las relaciones laborales, y la creación de nuevos empleos, lo que conlleva a la necesidad de replantear los temas laborales como son los modos de organización del trabajo y la productividad, generando necesarias reformas legislativas para resguardar los derechos laborales.

Como refieren Velásquez y Vera (2018:43) estos nuevos trabajos pueden ser ejecutados exclusivamente mediante la modalidad de teletrabajo; ejemplos claros de estas tendencias nuevas son las nuevas profesiones o puestos de trabajos como los Community Managers, quienes pueden realizar sus funciones desde cualquier lugar.

De acuerdo a Peralta et al, (2020: 334) es una actividad que cada día crece más en todo el mundo de la mano de los avances tecnológicos, como una respuesta a la globalización, que permite a la administración empresarial optimizar sus procesos y aumentar los beneficios. Sin dudas el teletrabajo como forma de prestación de servicios con el uso exclusivo de los medios tecnológicos, aceleró su implementación en América Latina a consecuencia de la pandemia originada por el covid-19, y su aplicación seguirá en desarrollo debido a los avances tecnológicos que se dan a nivel global y los beneficios que conllevan, aun cuando se requiere continuar su 
desarrollo a través de las legislaciones de cada estado de la región.

\section{Marco Jurídico del teletrabajo en Sudamérica}

contemplan dentro de su legislación nacional la figura jurídica del teletrabajo como una modalidad para la prestación de servicios, entre los cuales se destacan los que se describen en el siguiente cuadro 1:

\section{Cuadro 1}

Regulación del teletrabajo en Sudamérica antes del Covid-19

\begin{tabular}{lll}
\hline País & Legislación & Normativa \\
\hline \multirow{3}{*}{ Colombia } & $\begin{array}{l}\text { Artículo 2 Teletrabajo. Es una forma de organización laboral, que } \\
\text { Consiste en el desempeño de actividades remuneradas o prestación } \\
\text { Colombia (2008) }\end{array}$ & $\begin{array}{l}\text { de servicios a terceros utilizando como soporte las tecnologías de la } \\
\text { información y la comunicación - TIC para el contacto entre el trabajador } \\
\text { y la empresa, sin requerirse la presencia física del trabajador en un sitio } \\
\text { Lespecífico de trabajo. }\end{array}$ \\
\hline
\end{tabular}

Ministerio del Trabajo (2016) Acuerdo Ministerial No. MDT-2016-190. Ecuador.

Ecuador

Ministerio del Trabajo (2017) Acuerdo Ministerial No. MDT-20170090-A. Ecuador.
Art. 2. (...) El teletrabajo es una forma de prestación de servicios de carácter no presencial en jornadas ordinarias y especiales de trabajo a través de la cuales el trabajador/a realiza sus actividades fuera de las instalaciones del empleador, siempre que las necesidades y naturaleza del trabajo lo permitan, haciendo uso de las tecnologías de la información y comunicación (TIC), tanto para su gestión como para su administración y control.

Artículo 3: a) Teletrabajo: es la prestación de servicios de carácter no presencial en jornadas ordinarias y especiales de trabajo a través de la cual la o el servidor público realiza sus actividades fuera de las instalaciones de la institución pública para la cual labora, siempre que las necesidades y naturaleza del servicio lo permitan haciendo uso de las tecnologías de la información y comunicación TIC, tanto para su gestión como para su administración y control”.
Congreso de la República de

Perú Perú (2013) Ley No 30.036 de 2013.
Artículo 2. El teletrabajo se caracteriza por el desempeño subordinado de labores sin la presencia física del trabajador, denominado "teletrabajador", en la empresa con la que mantiene vínculo laboral, a través de medios informáticos, de telecomunicaciones y análogos, mediante los cuales se ejercen a su vez el control y la supervisión de las labores.

Art. 22. (...) Quedarán excluidos de la limitación de jornada de trabajo los trabajadores que presten servicios a distintos empleadores; los gerentes, administradores, apoderados con facultades de administración y todos aquellos que trabajen sin fiscalización superior inmediata; los contratados de acuerdo con este Código para prestar

Dirección del

Chile Trabajo (2019)

Código del

Trabajo. Chile. servicios en su propio hogar o en un lugar libremente elegido por ellos; los agentes comisionistas y de seguros, vendedores viajantes, cobradores y demás similares que no ejerzan sus funciones en el local del establecimiento. (...) Asimismo, quedan excluidos de la limitación de jornada, los trabajadores contratados para que presten sus servicios preferentemente fuera del lugar o sitio de funcionamiento de la empresa, mediante la utilización de medios informáticos o de telecomunicaciones. 


\section{Cont... Cuadro 1}

Congreso
Nacional
$(2011)$ Ley n
12.551/2011.
Brasilia.

Brasil

\begin{abstract}
Art. $6^{\circ}$. No se distingue entre el trabajo realizado en el establecimiento del empleador, el ejecutado en el domicilio del empleado y el realizado a distancia, siempre que estén caracterizados los supuestos de la relación de empleo.

Párrafo único. Los medios telemáticos e informatizados de mando, control y supervisión se equiparon, para fines de subordinación jurídica, a los medios personales y directos de mando, control y supervisión del trabajo ajeno.

Art. 75-B. Se considera teletrabajo la prestación de servicios preponderantemente fuera de las dependencias del empleador, con la utilización de tecnologías de información y de comunicación que, por su naturaleza, no se constituyan como trabajo externo. Párrafo único. La asistencia a las dependencias del empleador para la realización de actividades específicas que exijan la presencia del empleado en el establecimiento no des caracteriza el régimen de teletrabajo.
\end{abstract}

Congreso

Nacional (2017)

Ley $\mathrm{n}^{\circ} 13.467 / 17$.

Brasilia. aceptación del teletrabajo en Colombia se encuentran "el desconocimiento, seguido de la resistencia al cambio, los problemas del control del teletrabajador, el temor a la incertidumbre y la seguridad de la información. Otro de los aspectos que constituye una restricción para la implementación del teletrabajo es el acceso y uso de las tecnologías, ya que ésta juega un papel fundamental para su desarrollo, tal como lo refiere FloresCueto et al, (2020:507) cuando indican: El significativo incremento en el acceso $y$ uso de internet, ha generado (...) cambios trascendentes en la sociedad; actualmente se pueden desarrollar una serie de actividades en los entornos virtuales mediados por Tecnologías de Información y Comunicación como trabajar.

Al respecto, Culqui \& González (2016) y García (2013) las tecnologías de la información y comunicación son esenciales para las actividades económicas y en Perú, las normas que regulan el teletrabajo lo diferencia de otros modos laborales, por cuanto, las actividades de coordinación del trabajo, el control y supervisión por parte del 
empleador deben realizarse a través de las Tics. Tal es el caso que para Santillán (2020) ahora en tiempos de pandemia el uso de estas plataformas digitales ayuda al procesamiento de toda la información laboral, dentro de las cuales están involucradas las computadoras, internet, chat, el sistema zoom, correo electrónico.

En otros países, la puesta en marcha de estrategias para la implementación de esta alternativa laboral, ha generado mayores desafíos, puesto que sus formas de ejecución no se encontraban establecidas mediante Ley, aplicando de manera supletoria otras normas vigentes. Dentro de aquellos estados de la región que antes de la pandemia no contemplaban en sus normas el teletrabajo se encuentran los que se indican a continuación en el cuadro 2.

\section{Cuadro 2}

Normas supletorias para el teletrabajo en Sudamérica

\begin{tabular}{cl}
\hline País & \multicolumn{2}{c}{ Legislación } \\
\hline & \\
& \\
& Asamblea Nacional \\
& de la República \\
& Bolivariana de \\
Venezuela & Venezuela. (2012) \\
& Ley Orgánica \\
& del Trabajo, Los \\
& Trabajadores y Las \\
& Trabajadoras-
\end{tabular}

\begin{tabular}{|c|c|}
\hline & $\begin{array}{l}\text { Congreso Argentino } \\
\text { (1974) Ley del } \\
\text { Contrato de Trabajo }\end{array}$ \\
\hline Argentina & $\begin{array}{l}\text { Nro. } 20744 \text { del } \\
\text { 11/09/1974. Buenos } \\
\text { aires. }\end{array}$ \\
\hline
\end{tabular}

Artículo 204. Las modalidades especiales de condiciones de trabajo se establecerán en leyes especiales, elaboradas en corresponsabilidad y amplia participación de los sujetos de la relación laboral, particularmente los trabajadores, trabajadoras de cada modalidad y sus organizaciones sindicales.

Artículo 209. Trabajador o trabajadora a domicilio. Es toda persona que en su hogar o casa de habitación ejecuta un trabajo remunerado, con o sin ayuda de sus familiares, bajo la dependencia de uno o varios patronos o patronas, sin su supervisión directa, y utiliza para ello materiales e instrumentos propios, suministrados por el patrono o patrona o su representante, y está amparado por las disposiciones contenidas en el presente Capítulo. Estos trabajadores o trabajadoras gozan de los derechos relativos a la seguridad social.

Art. 21. - Contrato de trabajo. Habrá contrato de trabajo, cualquiera sea su forma o denominación, siempre que una persona física se obligue a realizar actos, ejecutar obras o prestar servicios en favor de la otra y bajo la dependencia de ésta, durante un período determinado o indeterminado de tiempo, mediante el pago de una remuneración. Sus cláusulas, en cuanto a la forma y condiciones de la prestación, quedan sometidas a las disposiciones de orden público, los estatutos, las convenciones colectivas o los laudos con fuerza de tales y los usos y costumbres.

ARTICULO $32^{\circ}$ Se entiende por trabajo a domicilio el que se realiza por cuenta ajena y con remuneración determinada, en el lugar de residencia del trabajador, en su taller doméstico o el domicilio del patrono. Se encuentran comprendidos dentro de esta definición: 1) Los que trabajan aisladamente o formando taller de familia en su domicilio, a destajo por cuenta de un patrono (...).
Congreso Nacional (1942) Ley General

Bolivia del 1942 . 08/12/1942. La Paz Bolivia.
Congreso de la Nación Paraguaya

Paraguay (1993) Ley $N^{\circ} 213 / 93$ que establece el Código de Trabajo.
Artículo $137^{\circ}$ : Trabajo a domicilio es toda labor por cuenta ajena ejecutada a jornal, por tarea o a destajo, en taller de familia, en el domicilio del trabajador o en otro lugar elegido por él, sin vigilancia o dirección inmediata del empleador o sus representantes.

Fuente: Legislaciones citadas 
Uno de los desafíos que dificultan la adecuada implementación del teletrabajo en Sudamérica es la falta de regulación jurídica, tal como se constata en el cuadro 2, como es el caso de Venezuela donde su aplicación está sometida a las normas previstas en la legislación laboral y la figura que más se asemeja al teletrabajo es el trabajo a domicilio. Al igual que Argentina, Bolivia y Paraguay que antes de la pandemia no estaba normado este modo de organización laboral en una Ley especial. En el caso de Uruguay no cuenta con un código de trabajo donde se encuentren recogidos las normas laborales, es decir la legislación laboral uruguaya está conformada por normativa Constitucional, Tratados Internacionales, leyes dispersas y un sin número de convenios colectivos.

Ahora bien, ante la emergencia sanitaria el mercado laboral se vio obligado a establecer las acciones necesarias para llevar a cabo el teletrabajo a fin de garantizar los derechos de los trabajadores y mantener los contratos laborales, lo que generó en consecuencia la necesaria promulgación de normas para respaldar las prácticas del teletrabajo en casa. Al respecto Buitrago (2020) afirma que, en América Latina, producto de la crisis, algunos estados han tomado la decisión de implementar en su legislación el teletrabajo como esa opción de continuidad de los negocios y la garantía de las condiciones laborales de los trabajadores. Para Santillán (2020) los gobiernos a nivel mundial motivaron a las empresas a valorar a sus trabajadores con el fin de prevenir el contagio por covid-19 y tomar algunas decisiones como la implementación del teletrabajo, seleccionando aquellos cargos admisibles para el desarrollar las actividades desde casa.
Al respecto Benavides (2020) refiere que, para garantizar que la pandemia finalice con el menor número de fallecidas se debe cumplir con la prevención de los riesgos del trabajo, en cuanto a la implementación de equipos de protección personal, y más aún en el control y monitoreo de los riesgos generados en las actividades realizadas bajo teletrabajo que afectan la salud física y mental.

\section{EI Teletrabajo y su regulación en Sudamérica frente al Covid-19}

El derecho laboral ha evolucionado de manera cambiante y permanente, conforme se transforman y actualizan los modos de producción, la economía, la industrialización y nuevas formas de contratación laboral; para enfrentar la crisis derivada del Covid-19 la evolución de las tecnologías, información y la comunicación han marcado el punto de partida en la implementación del teletrabajo.

En Colombia, La Presidencia de la República (2012) a través del Decreto No. 884 de 2012, reglamenta la Ley 1221 de 2008, que estableció los lineamientos para la implementación del teletrabajo. Guzmán y Abreo (2017:8) al referirse a esta Ley señalan que: "promueve el teletrabajo como una estrategia para el desarrollo de Ciudades Inteligentes y como mecanismo de inclusión para personas en condición de discapacidad". Esta Ley fue promulgada para cubrir ciertas necesidades en el campo laboral, en lugares específicos y con características determinadas. Al respecto Abril, Abril y Abril (2020) indican que en Colombia se han implementado grandes esfuerzos para regular el 
teletrabajo y documentar para que las empresas puedan contratar bajo este modo de trabajo.

Ante los efectos de la pandemia en Colombia el Ministerio del Trabajo (2020) emitió Circular Nro.0017 y Circular No. 0021 que establecen los parámetros para la promoción y prevención, así como, la atención de los casos contagiados por la enfermedad Covid-19, dejando previsto la obligación de empleadores con trabajadores provenientes de China que no presentaran síntomas respiratorios de implementar la estrategia del teletrabajo. De igual manera, indican las medidas de protección al empleo con ocasión de la fase de contención de Covid-19 y de la declaración de emergencia sanitaria, en la que refiere la implementación del trabajo en casa previsto en el artículo 6 numeral 4 de la Ley 1221, como circunstancia excepcional debido a la crisis sanitaria, de aplicación para todos los sectores económicos, señalando además que esta modalidad es diferente al teletrabajo.

En el Ecuador los avances tecnológicos, la globalización y las políticas adoptadas por el estado han abierto la posibilidad para que las empresas apliquen el teletrabajo, con fundamento a lo previsto en la Constitución de la República del Ecuador, 2008 que contempla en su artículo 33, el trabajo como un derecho y deber social, y el artículo 2 numeral 16 que establece el derecho que tienen las personas al acceso y el manejo de las tecnologías, información y comunicación (Sánchez, Montenegro y Medina, 2019).

El Ministerio del Trabajo (2016) emitió Acuerdo Ministerial No. MDT2016-190, que expide las normas que regulan el teletrabajo en el sector privado regulando la modalidad permanente en donde el trabajador realiza sus actividades siempre en un lugar distinto al del empleador y sólo cuando sea requerido podrá asistir al centro de trabajo y en la modalidad parcial ejecuta sus funciones fuera del lugar del empleador, hasta en un máximo de 24 horas semanales, y adicionalmente puede realizar su jornada en la empresa, el contrato es por escrito. Además, el artículo 3 requiere la descripción de las actividades que realizará el teletrabajador, el equipo de trabajo que empleará, las personas responsables en el suministro, instalación y mantenimiento; lo que indudablemente en el contexto del Covid-19, no fue posible, ya que de manera inesperada los trabajadores quedaron aislados en su domicilio realizando sus actividades en muchos casos con instrumentos de trabajo propios.

Aunado a lo anterior, mediante Acuerdo Ministerial No. MDT-2017 0090-A el Ministerio de Trabajo (2017) de Ecuador expide la Norma Técnica para regular el teletrabajo en el sector público, indicando además de lo previsto para el sector privado, la determinación del tiempo de ejecución del teletrabajo, la delimitación del lugar establecido para desarrollar las actividades y la determinación sobre la responsabilidad del estado en la provisión de bienes y servicios al teletrabajador.

Así pues, ante emergencia sanitaria, el Ministerio del Trabajo (2020) mediante Acuerdo Ministerial Nro. MDT- 2020-076, y Nro. MDT-2020080, dispone la implementación del teletrabajo emergente para el sector público y privado donde sólo debe variar el lugar de prestación de servicio, sin modificar las condiciones contractuales, y sin que se violenten los derechos, ni se originen causales para la terminación de la relación de trabajo, las autoridades en 
el sector público o los empleadores en el privado son los que deben establecer los lineamientos para el control y fiscalización de las actividades ejecutadas por el teletrabajador. Además, señala que bajo acuerdo los trabajadores y empleadores tomando en cuenta los efectos de la pandemia, pueden establecer convenios para aplicar modos de trabajos que atiendan a la situación de emergencia, prevención, y factores económicos resguardando el empleo y garantizando los derechos laborales.

En Perú el teletrabajo está regulado en la Ley 30.036, conjuntamente con el Reglamento de dicha Ley Decreto Supremo No. 017-2005, es considerado como una modalidad de trabajo especial que se caracteriza por el uso de las tecnologías aplicado en el sector público y privado, y se diferencia del trabajo a domicilio donde las actividades generalmente son manuales, ambos tienen su propia regulación (Valencia, 2018). Al referirse a esta Ley Culqui y González (2016) indican que el teletrabajo bajo esta norma comprende la ejecución de tareas bajo subordinación, sin la presencia del trabajador y con el uso de las tecnologías.

Ante los efectos generados por el Covid-19 el Ministerio del Trabajo y Promoción de Empleo (2020) emitió resolución Ministerial No. 055-20 $\mathrm{TH}$, donde aprueba la "Guía para la prevención del Coronavirus en el ámbito Laboral" que establece los lineamientos para la implementación del teletrabajo frente a la pandemia considerando que los empleadores y trabajadores pueden pactar de forma escrita el cambio de modalidad laboral presencial al teletrabajo de acuerdo a lo previsto en la legislación vigente, establecer los medios que requiere el trabajador para ejecutar sus labores, responsabilidad en la provisión de equipos, capacitación tecnológica, jornada de trabajo, salario, y el plazo que durará la prestación de servicio bajo teletrabajo.

Por su parte, en Argentina El Senado y la Cámara de Diputados (2020) para superar la pandemia y debido a la necesidad de regular de manera específica el teletrabajo, presentó para su aprobación el Proyecto de Ley de Regulación y Promoción del Teletrabajo aplicable para el sector privado y la Administración Pública, donde el teletrabajador realiza de forma total o parcial sus actividades dentro del domicilio o en un lugar distinto de las oficinas de la empresa, prevé el artículo 3 los principios que rigen el teletrabajo como son: la igualdad de trato, reversibilidad, derecho a la desconexión, privacidad, así como, derechos y obligaciones, requisitos del contrato, equipos, fiscalización, y responsabilidad.

En el caso de Argentina las limitaciones para adoptar el teletrabajo frente a la pandemia no sólo se suscriben a las tareas desarrolladas por el teletrabajador o al tipo de organización empresarial, por cuanto, en este contexto es necesario que los hogares estén dotados como mínimo de una conectividad a internet adecuada para poder implementar el teletrabajo (Albrieu, 2020).

En Chile según la Biblioteca del Congreso Nacional (2020) se sanciona la Ley 21220 que modifica el Código de Trabajo en materia de trabajo a distancia, incorporando de manera específica este modo de prestación de servicio, el cual podrá pactarse al inicio o en el desarrollo de la relación de trabajo, diferenciando el trabajo a distancia del teletrabajo, ya que en este último las actividades son ejecutadas a través del uso de herramientas tecnológicas, 
informáticas o de telecomunicaciones, donde la norma permite pactar la forma de desarrollar las tareas de acuerdo a las necesidades del trabajador, siempre que no se excedan los límites máximos de la jornada de trabajo, regula además los elementos que debe contener el contrato de trabajo, los materiales, equipos y herramientas para ejecutar las actividades, así como las medidas de salud y seguridad.

Bolivia ante la emergencia sanitaria la Presidencia Constitucional del Estado Plurinacional (2020) emitió Decreto Supremo No.4.218 donde dispuso que era necesario establecer una nueva forma de prestación de servicios no presencial en el domicilio del trabajador o en un lugar diferente, siempre que sea ajeno al del empleador y mediante el uso de las tecnologías, aplicable para el sector público y privado de forma temporal o permanente. Dicho Decreto prevé toda la normativa que regula el teletrabajo, como la jornada para su aplicación, las obligaciones del empleador o entidad pública, como son la provisión de equipos, capacitación al teletrabajador y medios de control, obligaciones del teletrabajador como el cumplimiento de las normas de seguridad, acceso al empleador sobre la información relativa al teletrabajo, y confidencialidad en la información.

En Venezuela la Presidencia de la República Bolivariana (2020) mediante Decreto No. 4198 declaró el estado de alarma para atender la emergencia sanitaria del coronavirus el cual prevé en su artículo 8 la posibilidad de suspensión de las actividades en determinadas zonas geográficas, además de la suspensión de las actividades laborales donde no sea posible la aplicación de la modalidad a distancia, por otra parte, el artículo 11 ordena la implementación de la educación a distancia en todo el territorio nacional.

El impulso del teletrabajo necesario por el covid-19, refleja la desigualdad en lo social, aspectos culturales, la educacional y capacidades tecnológicas en el mundo actual, este modo de trabajo presenta muchas barreras para los sectores vulnerables a diferencia de los países desarrollados (Domínguez, 2020).

En Brasil el Congreso Nacional (2017) a través de la Ley $n^{\circ} 13.467 / 17$ incorporó un capitulo con el fin de normar la relación laboral mediante la modalidad de teletrabajo, la cual reúne una serie de garantías y beneficios para generar más confianza al empleador y al teletrabajador que por mutuo acuerdo decidan pactar dicha modalidad de trabajo. En su arduo trabajo de frenar la propagación del coronavirus, según Atos do Poder Executivo (2020) la Presidencia de la República emitió la Medida Provisional $N^{\circ}$ 927, en el cual establece medidas laborales para hacer frente al estado de calamidad pública reconocido por el Decreto Legislativo $\mathrm{N}^{\circ} 6$, de 20 de marzo de 2020, donde dispone que los empleadores podrán optar por el teletrabajo, trabajo remoto o trabajo a distancia en las relaciones laborales bajo dependencia.

Uruguay ha venido aplicando esta modalidad de trabajo de forma atípica y voluntaria, no obstante, debido a la propagación de la enfermedad del coronavirus, La Presidencia de la República Oriental del Uruguay (2020) emitió Decreto $N^{\circ}$ 94/020 que pretende mitigar y prevenir las consecuencias de la propagación del virus Covid-19, el artículo 6, exhorta a todos los empleadores a la implementación y promoción del trabajo en el domicilio en aquellos casos que sea aplicable, 
estableciendo la obligación al empleador de informar a la Inspección General de Trabajo y suministrar al trabajador todo lo necesario para la ejecución de las labores.

De igual manera, Paraguay debido a la crisis económica y laboral, que se está viviendo a gran escala a nivel mundial, también ha visto la necesidad de incorporar en su ordenamiento jurídico el teletrabajo a través de la Ley $\mathrm{N}^{\circ}$ 6524, sancionada por el Congreso de la Nación Paraguaya (2020) que en su Artículo 20 establece la implementación del teletrabajo bajo relación de dependencia que ampara al sector público y privado, siempre que las actividades sean realizadas a distancia con el uso exclusivo de las tecnologías de la información y comunicación.

El importante avance y evolución de la figura jurídica del teletrabajo en Sudamérica alcanza su auge como alternativa para enfrentar el covid-19, países como Ecuador y Perú contemplan su regulación emergente mediante Acuerdos Ministeriales, en el caso de Argentina, Chile, Brasil y Bolivia el desarrollo ha sido hacia una regulación más específica y detallada en beneficio de los trabajadores y empleadores, Venezuela, Uruguay y Paraguay al no tener contemplado dentro de su legislación este modo de trabajo, debido a las medidas de aislamiento obligatorio para la prevención del coronavirus establecen la posibilidad de su implementación.

\section{Conclusiones}

La pandemia del covid-19 generó grandes cambios y transformaciones sociales a nivel global, uno de los cuales lo conforman los nuevos modos de prestación de servicios que dan impulso al desarrollo del teletrabajo a través del uso de las tecnologías de información y comunicación. La implementación del teletrabajo en Sudamérica iniciaba su desarrollo con una escasa regulación jurídica en los diferentes países, puesto que, a nivel legislativo la región no se encontraba preparada para la aplicación de este modo de organización laboral que aún se considera novedoso en muchos aspectos, sin embargo debido al impacto generado por el covid-19 en el mercado laboral fueron necesarios y urgentes los pronunciamientos de los Órganos Ministeriales, Poder Legislativo y hasta Decretos presidenciales para regular y desarrollar de la manera más idónea esta modalidad de prestación de servicios con el fin de amparar y resguardar el derecho al trabajo y los medios de producción en el sector público y privado.

A pesar de la evolución jurídica en algunos estados de la región, aún se encuentran barreras en la implementación del teletrabajo, tales como el efectivo acceso a la conectividad o internet, cambio de cultura y la aceptación del trabajo a distancia, remoto o teletrabajo.

No obstante, ante todas estas limitantes que puedan presentarse es fundamental el marco jurídico que regule las relaciones laborales desarrolladas en teletrabajo, donde se establezcan de manera expresa la jornada de trabajo, determinación del lugar de prestación de servicios, medidas de salud y seguridad laboral que deben ser adoptadas, medidas de control y monitoreo de las actividades, capacitación sobre el uso de herramientas y equipos tecnológicos, más aun ante el impacto de la pandemia que ha sacudido al mudo desestabilizando la salud, la economía y en gran medida el mercado laboral. 
Ramírez Velásquez, Joanna Carolina; Tapia Tapia, Héctor Eduardo; Vega Abad, Cesar Remigio; Villagómez, Martha

Teletrabajo en Sudamérica: Un desafío jurídico frente al Covid-19

A pesar de los grandes esfuerzos visualizados en la investigación, la implementación y desarrollo jurídico del teletrabajo en Sudamérica requiere de mayor estudio y precisión legislativa para garantizar no sólo el cumplimento de los derechos que le asisten al teletrabajador, sino también establecer sus obligaciones para resguardar y asegurar el efectivo cumplimiento de los objetivos planteados en el desarrollo de sus tareas, de modo pues, ante las regulaciones dadas para enfrentar la pandemia se asumen nuevos retos que determinarán el éxito de esta modalidad de trabajo en el desarrollo productivo empresarial.

\section{Referencias bibliográficas}

Abril, L., Abril, M., y Abril, S. (2020). Seguridad y salud en el trabajo en teletrabajo autónomo en Colombia. Signos. Investigación es Sistema de Gestión, 12(1), 83-101.

Academia Interamericana de Teletrabajo para América Latina y el Caribe. (2017). Primer Informe. Estado del Teletrabajo en América Latina y el Caribe. https://cidtt.org/wp-content/ uploads/2018/03/Estado-delTeletrabajo-en-Am\%C3\%A9ricaLatina.pdf

Albrieu, R. (2020). Evaluando las oportunidades y los límites del teletrabajo en Argentina en tiempos del covid-19. Programa de Desarrollo Económico. https://www.cippec.org/ wp-content/uploads/2020/04/Albrieuabril-2020-Oportunidades-y-limitesdel-teletrabajo-en-Argentin...-3.pdf

Asamblea Nacional de la República Bolivariana de Venezuela (2012). Ley Orgánica del Trabajo, los Trabajadores y las Trabajadoras.
Gaceta Oficial N ${ }^{\circ} 6.076$ Extraordinario del 7 de mayo de 2012. Venezuela. https://oig.cepal.org/sites/default/ files/2012 leyorgtrabajo ven.pdf

Atos do Poder Executivo (2020). Presidencia de la República de Brasil. Medida Provisional N ${ }^{\circ} 927$ de fecha 22 de marzo de 2020. https:// bit.ly/3uMKY3B

Benavides, F. (2020). La salud de los trabajadores y la Covid-19. Creative Commons. Archivos de prevención de Riesgos Laborales, 23(2). https:// doi.org/10.12961/aprl.2020.23.02.02

Benjumea-Arias, M., Villa-Enciso, E., y Valencia-Arias, J. (2016). Beneficios e impacto del teletrabajo en el talento humano. Resultados desde una revisión de literatura. Revista CEA, 2(4), 59-73.

Biblioteca del Congreso Nacional de Chile (2020). Ley No. 21220. Modifica el Código de Trabajo en materia de trabajo a distancia. Promulgación 24 de marzo de 2020. https://www.leychile.cl/ navegar?idNorma $=1143741$

Buitrago, D. (2020). Teletrabajo: una oportunidad en tiempos de crisis. Revista CES Derecho, 11(1), 1-2.

Congreso Argentino. (1974). Ley del Contrato de Trabajo Nro. 20744 del 11/09/1974. Buenos aires. Texto ordenado por Decreto 390/1976. https://www.ilo.org/dyn/travail/ docs $/ 979 /$ Ley $\% 20 \mathrm{~N} \% \mathrm{C} 2 \%$ B0 $\% 20$ 20.744.pdf

Congreso de Colombia (2008). Ley 1221. Diario Oficial No. 47.052 de 16 de julio de 2008. https://www.mintic. gov.co/portal/604/articles-3703 documento.pdf

Congreso de la Nación Paraguaya. (1993). Ley No. 213/93. Código del Trabajo. Gaceta Oficial, 29 de octubre 
de 1993, núm. 105 bis, págs. 1-30. http://www.sipi.siteal.iipe.unesco. org/sites/default/files/sipi normativa/ paraguay ley nro 213 1993.pdf

Congreso de la Nación Paraguaya. (2020). Ley No. 6524. Que declara estado de emergencia en todo el territorio de la República del Paraguay ante la emergencia declarada por la Organización Mundial de la Salud a causa del Covid-19 o Coronavirus y se establecen medidas administrativas, fiscales y financieras. https://www.bacn.gov.py/ archivos/9156/LEY6524.pdf

Congreso de la República del Perú. (2013). Ley 30.036 de 2013. Ley que regula el teletrabajo. Perú. https://bit. ly/327XXjO

Congreso Nacional. (1942). Ley General del Trabajo del 08 de diciembre de 1942. La Paz-Bolivia. https://bit. Iy/3d6JZ8r

Congreso Nacional. (2011). Ley $n^{\circ}$ 12.551/2011. Brasilia, 15 de diciembre de 2011. http://www. planalto.gov.br/ccivil 03/ ato20112014/2011/lei/l12551.html

Congreso Nacional. (2017). Ley $\mathrm{n}^{\circ}$ 13.467/17. Brasilia, 13 de julio de 2017. http://www.ilo.org/dyn/ natlex/natlex4.detail?p lang $=e s \& p$ is $n=105767 \& p$ count $=2 \& p$ classification $=01$

Culqui Fernández, A \& González Torres, A (2016). El Teletrabajo: Una innovadora forma de organización del trabajo, una herramienta de inclusión laboral y su regulación jurídica en Perú. Revista Derecho \& Sociedad. $N^{\circ}$ 46, pp. 95-109. http:// revistas.pucp.edu.pe/index.php/ derechoysociedad/article/view/18823

Dirección del Trabajo. (2019). Gobierno de Chile. Código del Trabajo. https://www.dt.gob.cl/portal/1626/

\section{articles-95516 recurso 2.pdf}

Domínguez, J. (2020). Entendiendo el teletrabajo. Departamento de Informática y Departamento de Postgrado. Universidad Politécnica Territorial del estado AraguaVenezuela.

El Senado y Cámara de Diputados. (2020). Proyecto de Ley. Regulación y Promoción del Teletrabajo del 27 de mayo de 2020. Argentina. https:// www4.hcdn.gob.ar/dependencias/ dsecretaria/Periodo2020/PDF2020/ TP2020/2350-D-2020.pdf

Fernández, L. (2019). Reflexiones sobre el teletrabajo en Colombia. Revista Legem, 5(1), 23-51. https://doi. org/10.15648/legem.1.2019.2331

Flores-Cueto, J., Hernández, R y GarayArgandoña, R. (2020). Tecnología de Información: acceso a internet y brecha digital en Perú. Revista Venezolana de Gerencia, 25(90), 504-527. https://doi.org/10.37960/ rvg.v25i90.32396

García, M. T. (2013). El rol de las tecnologías de la información y comunicación en la gestión del conocimiento: un desafío estratégico en el nuevo contexto empresarial. Revista De Ciencias Sociales, 19(2). https://doi.org/10.31876/rcs. v19i2.25625

Guzmán, A., y Abreo, C. (2017). Las habilidades del teletrabajo para la competitividad. Forum Empresarial, (2), 5-30.

Ministerio de Producción y Trabajo. (2019). Teletrabajo en Argentina: primer libro blanco nacional de buenas prácticas en teletrabajo, trabajo remoto y trabajo conectado. 1a edición ampliada. Ciudad Autónoma de Buenos Aires: Secretaría de Gobierno de Trabajo y Empleo. https://www.argentina.gob. 
Ramírez Velásquez, Joanna Carolina; Tapia Tapia, Héctor Eduardo; Vega Abad, Cesar

Remigio; Villagómez, Martha

Teletrabajo en Sudamérica: Un desafío jurídico frente al Covid-19

ar/sites/default/files/20191009 libro blanco de teletrabajo.pdf

Ministerio de Trabajo y Promoción de Empleo (2020) Resolución Ministerial 055-2020 TH. Lima, Perú. $\quad$ https://cdn.www.gob.pe/ uploads/document/file/545998/ doc04965720200309090043.pdf

Ministerio del Trabajo (2020). Circular No. 0017. Bogotá, Colombia 24 de febrero de 2020.

Ministerio del Trabajo (2020). Circular No. 0021. Bogotá, Colombia 17 de marzo de 2020.

Ministerio del Trabajo (2020). República del Ecuador. Indicadores Laborales. Disponible en:http://www.trabajo. gob.ec/wp-content/uploads/2020/10/ Cifras-29-de-octubre.pdf

Ministerio del Trabajo. (2016). República del Ecuador. Acuerdo Ministerial Número MDT-2016-190. http:// www.trabajo.gob.ec/wp-content/ uploads/2016/10/Acuerdo Teletrabajo WEB.pdf

Ministerio del Trabajo. (2017). República del Ecuador. Acuerdo Ministerial Número MDT-2017-0090-A. http:// www.trabajo.gob.ec/wp-content/ uploads/downloads/2017/11/AMMDT-2017-0090-A-1.pdf

Ministerio del Trabajo. (2020) República del Ecuador. Acuerdo Ministerial Número MDT-2020-080. https:// www.trabajo.gob.ec/wp-content/ uploads/2020/03/ACUERDOMINISTERIAL-Nro.-MDT-2020-080signed.pdf

Ministerio del Trabajo. (2020). República del Ecuador. Acuerdo Ministerial Número MDT-2020-076. http:// www.trabajo.gob.ec/wp-content/ uploads/2020/03/ACUERDO-MDT2020-076-TELETRABAJO.pdf

Organización Internacional del Trabajo
OIT (2016). Departamento de políticas sectoriales. Las dificultades y oportunidades del teletrabajo para los trabajadores y empleadores en los sectores de servicios de tecnología de la información y las comunicaciones (TIC) y financieros. Ginebra, 24-26 de octubre de 2016. https://www.ilo.org/wcmsp5/ groups/public/---ed dialogue/--sector/documents/publication/ wcms 531116.pdf

Organización Mundial de la Salud OMS (2020). La OMS declara que el nuevo brote de coronavirus es una emergencia de salud pública de importancia internacional. Ginebra/ Washington, 30 de enero de 2020. https://bit.ly/39ZPJPo

Peralta Beltrán, A., Bilous, A., Flores Ramos, C., \& Bombón Escobar, C. (2020). El impacto del teletrabajo y la administración de empresas. RECIMUNDO, 4(1), 326-335. http:// doi.10.26820/recimundo/4.(1). enero.2020.326-335

Presidencia Constitucional del Estado Plurinacional de Bolivia. (2020). Decreto Supremo No. 4.218 de fecha 14 de abril de 2020. https://www. lexivox.org/norms/BO-DS-N4218. xhtml

Presidencia de la República Bolivariana de Venezuela. (2020). Decreto No. 4.198 de fecha 12 de mayo de 2020. Gaceta Oficial de la República Bolivariana de Venezuela $\mathrm{N}^{\circ} 6.535$ Extraordinaria. http://www.inac.gob. ve/wp-content/uploads/2020/08/ GOE6535.pdf

Presidencia de la República de Colombia. (2012). Decreto No. 884 de 2012, que reglamenta la Ley 1221 de 2008. Bogotá, Colombia. http://www. suin-juriscol.gov.co/viewDocument. asp?ruta=Decretos $/ 1183842$

Presidencia de la República Oriental 
del Uruguay (2020). Decreto 94/020 de fecha 16 de marzo de 2020. https://medios.presidencia.gub. uy/legal/2020/decretos/03/cons min 19.pdf

Rodríguez, A. y D’Errico, J. (2017). Teletrabajadores: entre los discursos optimistas y los contextos precarizados. Una aproximación desde el caso argentino. Revista Colombiana de Sociología, 40(2), 47-66. https://doi.org/10.15446/rcs. v40n2.66384

Sánchez, G., Montenegro, A., y Medina, P. (2019). Teletrabajo una propuesta de innovación en productividad empresarial. Digital Publisher. No. 5.1. https://doi. org/10.33386/593dp.2019.5-1.133

Santillán, W. (2020) El Teletrabajo en el COVID-19. Número Especial Desafíos Humanos ante el COVID-19. CienciAmérica. 9(2). https://dialnet.unirioja.es/servlet/ articulo?codigo $=7746439$

Tapasco-Alzate, O. y García-Giraldo, J. (2020). Asociación entre posturas administrativas de directivos y su disposición hacía la adopción del teletrabajo. Información Tecnológica, 31(1), 149-160. http://dx.doi.org/10.4067/S0718$\underline{07642020000100149}$

Valencia, A. (2018). Aspectos regulatorios del teletrabajo en Perú: Análisis y perspectivas. Revista del Instituto de Ciencias Jurídicas de Puebla. A.C., 12(41), 203-226. https://bit.ly/3a5Q6rE

Velásquez, C., y Vera, M. (2017). Teletrabajo: Una revisión teórica sobre sus ventajas y desventajas. Investigatio. (10), 41-53. https:// revistas.uees.edu.ec/index.php/IRR/ article/view/194 